\title{
A Novel Antidiabetic Food Produced via Solid-State Fermentation of Tartary Buckwheat by L. plantarum TK9 and L. paracasei TK1501
}

\author{
Lei Feng ${ }^{\S}$, Yufeng Xie ${ }^{\S}$, \\ Chenmiao Peng, Yuxiaoxue \\ Liu and Haikuan Wang*
}

State Key Laboratory of Food Nutrition and Safety, College of Biotechnology, Tianjin University of Science and Technology, No. 29 of 13th Avenue, Tianjin, PR China

Received: 28 September 2017 Accepted: 15 May 2018

\footnotetext{
${ }^{5}$ These authors contributed equally to this work

*Corresponding author:

Phone: +862260601958;

Fax: +862260602298;

E-mail: hkwang@aliyun.com
}

\section{SUMMARY}

Diabetes is a chronic metabolic disease characterized by hyperglycaemia and a number of potential complications that significantly reduce the patient's quality of life. In this study, we produced an antidiabetic functional food from Tartary buckwheat fermented by Lactobacillus plantarum TK9 and L. paracasei TK1501. The results of an orthogonal experimental design indicated that the three factors with the largest effects on the growth of $L$. plantarum TK9 and L. paracasei TK1501 in solid-state fermentation (SSF) were in the order: water ratio >inoculum size >time of fermentation. Under the optimal fermentation conditions comprising a 1:1.5 water ratio, $24 \mathrm{~h}$ of SSF and a $10^{7} \mathrm{CFU} / \mathrm{g}$ inoculum, the Tartary buckwheat fermented by L. plantarum TK9 and L. paracasei TK1501 yielded viable probiotic counts of $(2.3 \pm 0.7) \cdot 10^{9}$ and $(3.3 \pm 0.4) \cdot 10^{\circ} \mathrm{CFU} / \mathrm{g}$, respectively. The nutritional potential, as well as antioxidant and antidiabetic properties of ethanolic extracts from fermented Tartary buckwheat were investigated. The highest a-glucosidase inhibitory activity, with an IC of $0.51 \mathrm{mg} / \mathrm{mL}$, was present in Tartary buckwheat fermented by L. plantarum TK9. However, Tartary buckwheat fermented by L. paracasei TK1501 had the highest dipeptidyl peptidase IV (DPP-IV) inhibition, with an $\mathrm{IC}_{50}$ of $2.47 \mathrm{mg} / \mathrm{mL}$. Therefore, fermentation by both $L$. plantarum TK9 and L. paracasei TK1501 has the potential to yield a product that can help regulate the levels of blood glucose as part of a diabetic diet.

Key words: Tartary buckwheat, fermentation, a-glucosidase inhibition, dipeptidyl peptidase IV inhibition, antidiabetic

\section{INTRODUCTION}

Diabetes constitutes a metabolic disorder characterized by chronic hyperglycaemia due to either insufficient insulin production (diabetes mellitus type 1) or insulin resistance (diabetes mellitus type 2) (1). It is predicted that the global number of type 2 diabetes patients will exceed 300 million by 2030 (2). Dietotherapy is the most basic therapy for diabetes, which makes the production of food suitable for diabetics especially important.

In recent years, there has been renewed interest in the utilization of Tartary buckwheat (Fagopyrum tataricum (L.) Gaertn.) due to its antihyperglycemic benefits (3). This antidiabetic effect of Tartary buckwheat is related to its unique chemical composition (4). It was discovered that Tartary buckwheat possesses higher concentrations of certain bioactive phytochemicals than common buckwheat (5). Moreover, the total dietary fibre content of Tartary buckwheat seeds was found to be $26 \%$, with $0.54 \%$ soluble and $24 \%$ insoluble fibre (6). Furthermore, Tartary buckwheat is an important source of phenolic acids and flavonoids, including $p$-hydroxybenzoic, protocatechuic, caffeic, chlorogenic, gallic, ferulic, $p$-coumaric, syringic and vanillic acids (7). Flavonoids are the most crucial component of total buckwheat polyphenols as well as the most important health-promoting factors. In Tartary buckwheat seeds, rutin is the major flavonoid, followed by quercetin (8). Flavonoid compounds have remarkable antioxidant effects, providing various health benefits such as antihypertensive, antidiabetic and anticancer effects (9). Tartary buckwheat is an excellent source of these phytochemicals, and it is in fact bitter due to its large flavonoid content. 
Diabetes has become a major disease worldwide, and it can lead to serious complications, causing enormous damage to the quality of life and health. The main target in the treatment of diabetes is the control of blood glucose levels (10). Several contemporary classes of hypoglycaemic agents seem to provide an effective treatment for diabetes (11). Among them, inhibitors of dipeptidyl peptidase IV (DPP-IV) are becoming a hot topic in the development of new hypoglycaemic drugs (12). The glucagon-like peptide-1 (GLP-1) can have a longer half-life due to DPP-IV inhibition, and reach the threshold of glucagon homeostasis (1). A number of synthetic DPP-IV inhibitors have shown promising results in the treatment of diabetes type 2 (13). The a-glucosidase inhibitors, being the best-tolerated antidiabetics, have the main effect of reducing a-glucosidase in the microvilli in order to delay the absorption of glucose and fructose, lower the postprandial blood glucose spikes and reduce the requirement for insulin injections $(12,14)$. However, such drugs have side effects, such as flatulence and diarrhoea, and there is a demand for fermented food with DPP-IV inhibitory and a-glucosidase inhibitory properties for the oral treatment of diabetes type 2 patients (15).

SSF has recently received much interest as an alternative to the more costly submerged fermentation (SmF) because of its potential to convert inexpensive agro-industrial solid residues and plant material into a variety of valuable products (16). This research uses Tartary buckwheat as a substrate for SSF of probiotics, with the aim to increase the contents of functional components as well as the nutritional and health-promoting value of the final product by complex metabolic reactions of microorganisms. Therefore, the objective of this study is to evaluate the effect of SSF on the chemical composition of Tartary buckwheat, as well as on its antioxidant and antihyperglycaemic activities.

\section{MATERIALS AND METHODS}

\section{Microorganisms and materials}

The microorganisms were deposited in the China General Microbiological Culture Collection Center (CGMCC) in Beijing. The CGMCC accession number of Lactobacillus plantarum TK9 is 11891 , and that of $L$. paracasei TK1501 is 13130.

The Tartary buckwheat samples used in this study were grown in Kunming, Yunnan province, PR China, and collected in October 2016. The grains were cleaned and stored in the dark in polyethylene containers at room temperature for less than 3 months.

\section{Preparation of microbiological cultures}

Lactobacillus plantarum TK9 and L. paracasei TK1501 were activated in $10 \mathrm{~mL}$ of De Man, Rogosa and Sharpe (MRS) broth (Oxoid, Basingstoke, UK) at $37^{\circ} \mathrm{C}$ for $18 \mathrm{~h}$, using $1 \%$ inocula. The $A_{600 \mathrm{~nm}}$ of the resulting culture of $L$. plantarum TK9 was between 1.0 and 1.2 , with viable counts of $(3.5 \pm 0.4) \cdot 10^{9} \mathrm{CFU} / \mathrm{mL}$; and the $A_{600 \mathrm{~nm}}$ of L. paracasei TK1501 was between 1.4 and 1.6, with viable counts of $(5.5 \pm 0.4) \cdot 10^{9} \mathrm{CFU} / \mathrm{mL}$. The cultures were centrifuged (GL20A, Xiangyi, Hunan, PR China) at $5000 \times g$ for 10 min, the supernatants were discarded, and the bacterial cells resuspended in sterile saline solution and adjusted to $10^{9} \mathrm{CFU} /$ $\mathrm{mL}$. Thus obtained suspensions were applied as inocula for SSF.

\section{Optimization of fermentation conditions using orthogonal experimental design}

Table 1 shows the influence factors and level values selected in this study. The orthogonal design helped to analyze the performance of the fermented Tartary buckwheat and determine the level of influence of factors (water ratio, inoculum size, time) affecting the total viable counts of the probiotic bacteria.

Table 1. Levels and factors affecting the solid-state fermentation (SSF) of Tartary buckwheat (TBW)

\begin{tabular}{cccccc} 
Independent variable & Factor & \multicolumn{5}{c}{ Level } \\
\cline { 3 - 7 } & & 1 & 2 & 3 & 4 \\
$\frac{m(\mathrm{TBW})}{V(\text { water })}$ & $\mathrm{A}$ & $1: 1.5$ & $1: 1.25$ & $1: 1$ & $1: 0.75$ \\
$N($ inoculum $) /(\mathrm{CFU} / \mathrm{g})$ & $\mathrm{B}$ & $5 \cdot 10^{6}$ & $10^{7}$ & $5 \cdot 10^{7}$ & $10^{8}$ \\
$t / \mathrm{h}$ & $\mathrm{C}$ & 12 & 24 & 36 & 48 \\
\hline
\end{tabular}

Tartary buckwheat grain substrate $(40 \mathrm{~g})$ and sterile distilled water were mixed in conical flasks ( $250 \mathrm{~mL}$; Deschem, Changshu, PR China) in mass per volume ratios as shown in Table 2 and Table 3, autoclaved (SX-500; Tomy, Fukushima, Japan) at $121^{\circ} \mathrm{C}$ for $20 \mathrm{~min}$, and cooled to $37^{\circ} \mathrm{C}$ before the addition of the required amounts of $L$. plantarum TK9 or L. paracasei TK1501 starter cultures. Fermentation of the inoculated substrates occurred at $37^{\circ} \mathrm{C}$ in an incubator (SHKE6000-1CE; Thermo Fisher Scientific, Waltham, MA, USA). Afterwards, samples from the optimal combinations were freeze-dried using a DW3 freeze dryer ( $\mathrm{He}-$ to-Holten A/S; Allerød, Denmark) and stored at $-20{ }^{\circ} \mathrm{C}$ for further analysis. The native unfermented samples (inoculated with the same volume of sterile saline) collected at $0 \mathrm{~h}$ were used as the negative control. SSF was performed in triplicate. SPSS software v. 22.0 (17) was used to perform the statistical analysis.

\section{Determination of the total viable counts}

Total viable counts of bacterial cells were assessed according to the method described by Zhang et al. (18). The fermented Tartary buckwheat $(10 \mathrm{~g})$ was homogenized for $15 \mathrm{~s}$ in $90 \mathrm{~mL}$ of sterilized physiological saline (0.85\%) in a blender (JT-C; Jintian, Luohe, PR China). Total viable counts of L. plantarum TK9 and $L$. paracasei TK1501 were made using a pour plate method and MRS agar (Oxoid) after serial dilution in maximum recovery diluents. Serial dilutions were prepared in sterilized physiological saline and $1 \mathrm{~mL}$ of the appropriate dilution was poured on plates in triplicate. The poured plates of L. plantarum TK9 were incubated at $37^{\circ} \mathrm{C}$ for $(48 \pm 2) \mathrm{h}$. The cultures of $L$. paracasei TK1501 were incubated at $37^{\circ} \mathrm{C}$ for $(60 \pm 2) \mathrm{h}$. The colonies were then counted, and the viable counts were expressed as colony forming units per gram (CFU/g) of the sample. 
Table 2. Orthogonal experimental design of different factor levels (values 1-4): the experimental results for factors A, B, C with the measured Tartary buckwheat+L. plantarum TK9

\begin{tabular}{|c|c|c|c|c|}
\hline $\begin{array}{c}\text { Experiment } \\
\text { no. }\end{array}$ & $\begin{array}{c}\text { Water ratio } \\
\text { (A) }\end{array}$ & $\begin{array}{l}\text { Inoculum } \\
\text { size (B) }\end{array}$ & Time (C) & $\begin{array}{l}N(\text { viable } \\
\text { count })^{*} \\
(\text { CFU/g) }\end{array}$ \\
\hline 1 & 1 & 1 & 1 & $1.86 \cdot 10^{9}$ \\
\hline 2 & 1 & 2 & 2 & $2.26 \cdot 10^{9}$ \\
\hline 3 & 1 & 3 & 3 & $1.95 \cdot 10^{9}$ \\
\hline 4 & 1 & 4 & 4 & $1.67 \cdot 10^{9}$ \\
\hline 5 & 2 & 1 & 2 & $2.00 \cdot 10^{9}$ \\
\hline 6 & 2 & 2 & 1 & $2.17 \cdot 10^{9}$ \\
\hline 7 & 2 & 3 & 4 & $1.72 \cdot 10^{9}$ \\
\hline 8 & 2 & 4 & 3 & $1.67 \cdot 10^{9}$ \\
\hline 9 & 3 & 1 & 3 & $1.76 \cdot 10^{9}$ \\
\hline 10 & 3 & 2 & 4 & $1.60 \cdot 10^{9}$ \\
\hline 11 & 3 & 3 & 1 & $1.72 \cdot 10^{9}$ \\
\hline 12 & 3 & 4 & 2 & $1.89 \cdot 10^{9}$ \\
\hline 13 & 4 & 1 & 4 & $1.46 \cdot 10^{9}$ \\
\hline 14 & 4 & 2 & 3 & $1.85 \cdot 10^{9}$ \\
\hline 15 & 4 & 3 & 2 & $1.62 \cdot 10^{9}$ \\
\hline 16 & 4 & 4 & 1 & $1.43 \cdot 10^{9}$ \\
\hline $\mathrm{K}_{1}$ & 7.74 & 7.08 & 7.18 & \\
\hline $\mathrm{K}_{2}$ & 7.56 & 7.88 & 7.77 & \\
\hline $\mathrm{K}_{3}$ & 6.97 & 7.01 & 7.23 & \\
\hline $\mathrm{K}_{4}$ & 6.36 & 6.66 & 6.45 & \\
\hline $\mathrm{k}_{1}$ & 1.935 & 1.77 & 1.795 & \\
\hline $\mathrm{k}_{2}$ & 1.89 & 1.97 & 1.9425 & $A>C>B$ \\
\hline $\mathrm{k}_{3}$ & 1.7425 & 1.7525 & 1.8075 & \\
\hline $\mathrm{k}_{4}$ & 1.59 & 1.665 & 1.6125 & \\
\hline
\end{tabular}

*Each value in the table represents the mean of $N=3, \mathrm{~K}_{\mathrm{i}}=$ the sum of the corresponding test results when the level factor is i for any factor, $\mathrm{k}_{\mathrm{i}}=\mathrm{K}_{\mathrm{i}} / 4$ (4 is the number of levels)

\section{Preparation of extracts}

The freeze-dried samples from the optimal combination in the orthogonal experiment and the negative control were ground to a powder using an M20 universal mill (IKA, Staufen, Germany). Then, $10 \mathrm{~g}$ of the freeze-dried SSF powder were extracted with $200 \mathrm{~mL}$ of $70 \%$ (by volume) ethanol for $2 \mathrm{~h}$ in an ultrasonic extractor (KH-600TDV; Hechuang, Kunshan, PR China). Afterwards, the samples were centrifuged (Heraeus $^{\mathrm{TM}}{ }$, Multifuge ${ }^{\mathrm{TM}} \mathrm{X} 1 \mathrm{R}$; Thermo Fisher Scientific) at $25155 \times \mathrm{g}$ and $4{ }^{\circ} \mathrm{C}$ for $10 \mathrm{~min}$, and the supernatants were collected. The residue was then suspended in $100 \mathrm{~mL}$ of $70 \%$ (by volume) ethanol, ultrasonicated and centrifuged under the same conditions. The supernatants were combined, filtered through Whatman no. 1 paper (GE Healthcare, Chicago, IL, USA) and freeze-dried. An aliquot comprising $5 \mathrm{mg}$ of the freeze-dried sample was stored at $-20^{\circ} \mathrm{C}$ and dissolved in $1 \mathrm{~mL}$ of phosphate buffer $(0.1 \mathrm{M}, \mathrm{pH}=6.8$; Sinopharm Chemical Reagent Co., Ltd, Shanghai, PR China) immediately before analysis.

\section{Determination of the total phenolic content}

The total phenolic content (TPC) in the extracts was determined by a modified Folin-Ciocalteu method (19). Briefly, $100 \mu \mathrm{L}$ of each diluted extract were mixed with $500 \mu \mathrm{L}$ of
Table 3. Orthogonal experimental design of different factor levels (values 1-4): the experimental results for factors A, B, C with the measured Tartary buckwheat+L. paracasei TK1501

$\begin{array}{ccccc}\begin{array}{c}\text { Experiment } \\ \text { no. }\end{array} & \begin{array}{c}\text { Water ratio } \\ (\mathrm{A})\end{array} & \begin{array}{c}\text { Inoculum } \\ \text { size }(\mathrm{B})\end{array} & \text { Time }(\mathrm{C}) & \begin{array}{c}\text { N(viable } \\ \text { count })^{*}\end{array} \\ \text { (CFU/g) }\end{array}$

*Each value in the table represents the mean of $N=3$

Folin-Ciocalteu reagent (Sangon Biotech, Shanghai, PR China) and $6 \mathrm{~mL}$ of distilled water, and shaken for $1 \mathrm{~min}$. Afterwards, 2 $\mathrm{mL}$ of a $15 \%$ (by mass per volume) $\mathrm{Na}_{2} \mathrm{CO}_{3}$ (Sinopharm Chemical Reagent Co., Ltd, Shanghai, PR China) solution were added to the mixture, shaken once again for $2 \mathrm{~min}$, and the volume of the solution was adjusted to $10 \mathrm{~mL}$ with distilled water. Finally, the mixture was incubated in the dark for $2 \mathrm{~h}$ at room temperature. The absorbance at $750 \mathrm{~nm}$ was recorded on an InfiniteM200 PRO multifunctional microplate reader (Tecan, Männedorf, Switzerland) against a solution without sample as blank (100 $\mu \mathrm{L}$ of $70 \%$ ethanol instead of the test samples). A standard curve was prepared using gallic acid (Sigma-Aldrich, St. Louis, MO, USA):

$$
A=0.4789 c \pm 0.0303
$$

where $A$ is the absorbance at $750 \mathrm{~nm}$ and $c$ is the concentration of gallic acid $\left(c=0.2-1 \mathrm{mg} / \mathrm{mL}, \mathrm{R}^{2}=0.9978\right)$.

Samples were independently analyzed in triplicate and the TPC was expressed as milligrams of gallic acid equivalents (GAE) per gram of extract.

\section{Determination of total flavonoid content}

Determination of total flavonoid content (TFC) followed a published colorimetric method (20) with slight modifications 
as follows: the mixture contained $0.3 \mathrm{~mL} \mathrm{NaNO}_{2}$ (Damao Chemical Reagent Factory, Tianjin, PR China) solution (5\%, by mass per volume), $0.6 \mathrm{~mL} \mathrm{AlCl}_{3}$ (Sinopharm Chemical Reagent Co., Ltd) solution (10\%, by mass per volume), $2 \mathrm{~mL} \mathrm{NaOH}$ (Sinopharm Chemical Reagent Co., Ltd) solution (1 M) and $1 \mathrm{~mL}$ diluted sample solution. The volume of the mixture was adjusted to $10 \mathrm{~mL}$ with distilled water. After $5 \mathrm{~min}$ of incubation at room temperature, the absorbance at $507 \mathrm{~nm}$ was recorded with multifunctional microplate reader (InfiniteM200 PRO; Tecan) against the mixture without sample solution as a blank. The TFC was expressed as rutin (Yuanye Biological Technology, Shanghai, PR China) equivalents per g of sample using the calibration curve of rutin:

$$
A=0.636 c \pm 0.0388
$$

where $A$ is the absorbance at $507 \mathrm{~nm}$ and $c$ is the concentration of rutin $\left(R^{2}=0.9974\right)$.

\section{Determination of a-glucosidase inhibition}

The a-glucosidase inhibition by the samples was assessed according to the method described by Zeng et al. (12) with slight modifications. Briefly, the reaction mixture contained $25 \mu \mathrm{L}$ of $10 \mathrm{mM} p$-nitrophenyl-a-D-glucopyranoside (PNPG; Sigma-Aldrich) and $25 \mu \mathrm{L}$ of the sample preincubated at 37 ${ }^{\circ} \mathrm{C}$ for $10 \mathrm{~min}$. The reaction was initiated by the addition of 50 $\mu \mathrm{L}$ a-glucosidase solution $(0.16 \mathrm{U} / \mathrm{mL}$; Sigma-Aldrich) diluted with $0.1 \mathrm{M}$ phosphate buffer, and incubated at $37^{\circ} \mathrm{C}$ for 30 min. The reaction was terminated by adding $100 \mu \mathrm{L}$ of $0.1 \mathrm{M}$ $\mathrm{Na}_{2} \mathrm{CO}_{3}$ (Sigma-Aldrich). The enzymatic activity was quantified based on the measurements of the absorbance of the samples at $405 \mathrm{~nm}$ on a Multiskan MK3 plate reader (Thermo Fisher Scientific). Each test sample was analyzed in technical triplicate, and the absorbance values were corrected against sample blanks in which a-glucosidase was replaced with phosphate buffer. The positive control (a-glucosidase activity with no inhibitor) and negative control (no a-glucosidase activity) were prepared by using phosphate buffer instead of the sample or instead of the sample and the a-glucosidase solution, respectively. The a-glucosidase inhibition rate was calculated as follows:

Inhibition $=\left(1-\frac{A(\text { sample })-A(\text { blank })}{A(\text { positive control })-A(\text { negative control })}\right) \cdot 100 \quad / 3 /$

\section{Determination of DPP-IV inhibition}

Dipeptidyl-peptidase IV (DPP-IV) inhibitory activity was assessed according to the method described by Zeng et al. (12) with some modifications. Briefly, $25 \mu \mathrm{L}$ Gly-Pro- $p$-nitroanilide ( $6 \mathrm{mM}$; Sigma-Aldrich) and $25 \mu \mathrm{L}$ Tartary buckwheat sample, or $25 \mu \mathrm{L}$ phosphate buffer saline (PBS; Sigma-Aldrich) as a control, were mixed and preincubated at $37^{\circ} \mathrm{C}$ for $10 \mathrm{~min}$. The reaction was initiated by adding $50 \mu \mathrm{L}$ DPP-IV from porcine kidney $\left(3 \cdot 10^{-4} \mathrm{U} / \mathrm{L}, \geq 10 \mathrm{U} / \mathrm{mg}\right.$ protein; Sigma-Aldrich) and the mixture was incubated at $37^{\circ} \mathrm{C}$ for $60 \mathrm{~min}$. The reaction was terminated by adding $100 \mu \mathrm{L}$ of $1 \mathrm{M}$ sodium acetate buffer ( $\mathrm{pH}=4.0$; Sinopharm Chemical Reagent Co., Ltd), and the absorbance of the samples at $405 \mathrm{~nm}$ was measured on a Multiskan MK3 plate reader (Thermo Fisher Scientific). Each sample was analyzed in technical triplicate, and the absorbance values were normalized to sample blanks in which DPP-IV was replaced with Tris- $\mathrm{HCl}$ buffer $(0.1 \mathrm{M}, \mathrm{pH}=8.0$; Solarbio, Beijing, PR China). The negative control (no DPP-IV activity) and positive control (DPP-IV activity with no inhibitor) were prepared by using Tris- $\mathrm{HCl}$ buffer $(100 \mathrm{mM}, \mathrm{pH}=8.0$; Solarbio) instead of the sample or instead of the DPP-IV solution and the sample, respectively. Diprotin A (Sigma-Aldrich) was used as a standard inhibitor. The DPP-IV inhibition rate was calculated using Eq. 3.

\section{RESULTS AND DISCUSSION}

\section{Statistical analysis of the results obtained using orthogonal experimental design}

The most important factors for consideration in the development of functional food are the bioactive components. In this study, we developed a probiotic product in a solid-state fermentation on Tartary buckwheat, taking into consideration the final viable probiotic cell count. Thus, the fermentation parameters were optimized in order to obtain a high growth rate of the lactic acid bacteria (LAB) in Tartary buckwheat. We designed an orthogonal experiment with three factors and four levels (Table 1). The results served to find the optimal fermentation conditions and analyze the relationship between the factors and systemic performance of the fermentation (Table 2 and Table 3). $\mathrm{K}_{\mathrm{i}}$ represents the sum of the corresponding test results when the level number is i for any factor (Table 3), and $\mathrm{k}_{\mathrm{i}}=\mathrm{K}_{\mathrm{i}} / 4$ ( 4 is the number of levels). The larger the $R$ value $\left(R=K_{\max }-K_{\min }\right)$, the greater the influence of this factor on the viable count. We found that the $R$ values of the water ratio (A), inoculum size $(B)$ and time of fermentation $(C)$ for TK9 and TK1501 were $R=0.345,0.305,0.330$ and $R=0.6575$, $0.4375,0.5025$, respectively. The effect of the factors on the number of viable bacteria was in the order: $A>C>B$. Thus, the optimal fermentation conditions were a 1:1.5 water ratio, a 24-hour fermentation period and $10^{7} \mathrm{CFU} / \mathrm{g}$ inoculum size (A1C2B2). Subsequently, analysis of variance (ANOVA) determined the significance of the model (Table 4 and Table 5). The $p$-values served as a tool to check the significance of each coefficient; the lower the $p$-value, the higher the significance of the corresponding coefficient. The corresponding $\mathrm{p}<0.05$ suggested that the water ratio, inoculum size and time of fermentation are significant terms. Therefore, a small variation of their values will notably alter the total viable count. Under the optimal conditions, the highest viable counts of Tartary buckwheat fermented by L. plantarum TK9 and L. paracasei TK1501 were $(2.3 \pm 0.7) \cdot 10^{9} \mathrm{CFU} / \mathrm{g}$ and $(3.3 \pm 0.4) \cdot 10^{9} \mathrm{CFU} / \mathrm{g}$, respectively. 
Table 4. Analysis of variance and regression analysis for L. plantarum TK9

\begin{tabular}{lccccc} 
Source & SS & df & Mean square & $F$ & $P$ \\
Water ratio (A) & 0.293 & 3 & 0.098 & 7.005 & 0.02 \\
Inoculum size (B) & 0.199 & 3 & 0.066 & 4.763 & 0.05 \\
Time (C) & 0.220 & 3 & 0.073 & 5.266 & 0.04 \\
Error & 0.084 & 6 & 0.014 & & \\
Corrected total & 0.796 & 15 & & & \\
\hline
\end{tabular}

$\mathrm{SS}=$ sum of squares, $\mathrm{F}=$ variance ratio, $\mathrm{P}=$ probability

Table 5. Analysis of variance and regression analysis for L. paracasei TK1501

\begin{tabular}{lccccc} 
Source & SS & df & Mean square & $F$ & $P$ \\
Water ratio (A) & 0.873 & 3 & 0.291 & 9.785 & 0.01 \\
Inoculum size (B) & 0.434 & 3 & 0.145 & 4.870 & 0.48 \\
Time (C) & 0.492 & 3 & 0.197 & 6.631 & 0.25 \\
Error & 0.178 & 6 & 0.030 & & \\
Corrected total & 2.077 & 15 & & & \\
\hline
\end{tabular}

$\mathrm{SS}=$ sum of squares, $\mathrm{F}=$ variance ratio, $\mathrm{P}=$ probability

\section{Total phenolic content during Tartary buckwheat fermentation}

During the fermentation of Tartary buckwheat by $L A B$, the composition of the buckwheat changes under the influence of bacterial metabolism. Hence, fermentation affected the bioactive constituents (Table 6). In Tartary buckwheat extracts, TPC (expressed as GAE) increased from $(243.0 \pm 14.0) \mathrm{mg} / \mathrm{g}$ in the dry extract of native unfermented sample to $(251.8 \pm 10.4) \mathrm{mg} / \mathrm{g}$ in the extract of Tartary buckwheat fermented by L. plantarum TK9. The TPC of Tartary buckwheat fermented by L. paracasei TK1501 was $(241.5 \pm 5.4) \mathrm{mg} / \mathrm{g}$ dry extract, without significant changes compared to the native unfermented material. Zhu (5) and Ran and Ma (21) reported a similar TPC in Tartary buckwheat. The possible explanation for these results is that the metabolic activity of microbes can modify the levels of bioactive compounds during fermentation (22). Some reports stated that fermentation by L. plantarum is an efficient process for increasing the concentration of phenolic compounds in fermented cowpea flour $(23,24)$. The $\beta$-glucosidase enzyme produced during fermentation is thought to catalyze the hydrolysis of complex polyphenols to yield simpler and biologically more active compounds, leading to an increase of the total phenolic content (25). In addition, some reports suggested that the higher antioxidant activity might be due to the presence of phenolic components (26).

Table 6. The chemical composition of native unfermented and fermented Tartary buckwheat (TBW)

\begin{tabular}{ccc} 
Sample & TPC(as GAE) $/(\mathrm{mg} / \mathrm{g})$ & $\mathrm{TFC} /(\mathrm{mg} / \mathrm{g})$ \\
Native TBW & $243.0 \pm 14.0$ & $25.5 \pm 1.1$ \\
$\begin{array}{c}\text { TBW+L. plantarum } \\
\text { TK9 }\end{array}$ & $251.8 \pm 10.4$ & $25.6 \pm 1.4$ \\
$\begin{array}{c}\text { TBW+L. paracasei } \\
\text { TK1501 }\end{array}$ & $241.5 \pm 5.4$ & $25.7 \pm 0.4$ \\
\hline
\end{tabular}

TPC=total phenolic content, TFC=total flavonoid content. Each value in the table represents the mean \pm S.D., $N=3, p<0.05$

\section{Total flavonoid content during Tartary buckwheat} fermentation

The TFC of the different samples is shown in Table 6. The TFC of the native unfermented Tartary buckwheat sample, as well as the samples fermented by $L$. plantarum TK9 and $L$. paracasei TK1501 was (25.5 \pm 1.1$),(25.6 \pm 1.4)$ and $(25.7 \pm 0.4) \mathrm{mg} / \mathrm{g}$, respectively. No significant differences in the TFC were observable between the native unfermented Tartary buckwheat and the preparations fermented by $L$. plantarum TK9 and $L$. paracasei TK1501. Importantly, this also means that there was no loss of TFC during fermentation. Moreover, recent reports stated that the TFC of Tartary buckwheat is much higher than that of common buckwheat $(12,27)$.

\section{Inhibition of a-glucosidase activity}

Diabetes is a chronic metabolic disorder mainly characterized by high blood glucose levels. Therefore, treatment of diabetes mainly focuses on reducing the fluctuations of blood glucose in order to reduce the subsequent complications. This has led to the development of a-glucosidase inhibitors as oral anti-diabetic agents, widely used in treatment of diabetes, that can postpone the digestion and absorption of carbohydrates, and thus reduce postprandial hyperglycaemia (28). In this study, the extracts of Tartary buckwheat fermented using the two different strains were able to inhibit a-glucosidase in a concentration-dependent manner (Fig. 1). All tested Tartary buckwheat extracts at a concentration of $1 \mathrm{mg} / \mathrm{mL}$ showed a-glucosidase inhibitory activities ranging from 29.25 to 75.82 $\%$, with the higher value obtained using Tartary buckwheat fermented by L. plantarum TK9. This extract at 0.008, 0.04, 0.1, $0.2,0.4,0.8$ and $1.0 \mathrm{mg} / \mathrm{mL}$ displayed a-glucosidase inhibitory activities of $(0.03 \pm 0.02),(0.3 \pm 0.2),(2.3 \pm 0.4),(20.2 \pm 1.6)$, $(42.8 \pm 0.3),(68.91 \pm 0.05)$ and $(75.8 \pm 0.2) \%$, respectively, with an $I_{50}$ value of $0.51 \mathrm{mg} / \mathrm{mL}$. By contrast, the extract of native Tartary buckwheat at the same concentrations had inhibitory activities of (1.5 \pm 7.8$),(4.9 \pm 1.6),(5.7 \pm 4.0),(17.8 \pm 2.2),(22.6 \pm 1.5)$, $(44.1 \pm 1.3)$ and $(60.5 \pm 0.6) \%$, respectively, with an $I C_{50}$ value $0.87 \mathrm{mg} / \mathrm{mL}$. The extract fermented with L. paracasei TK1501

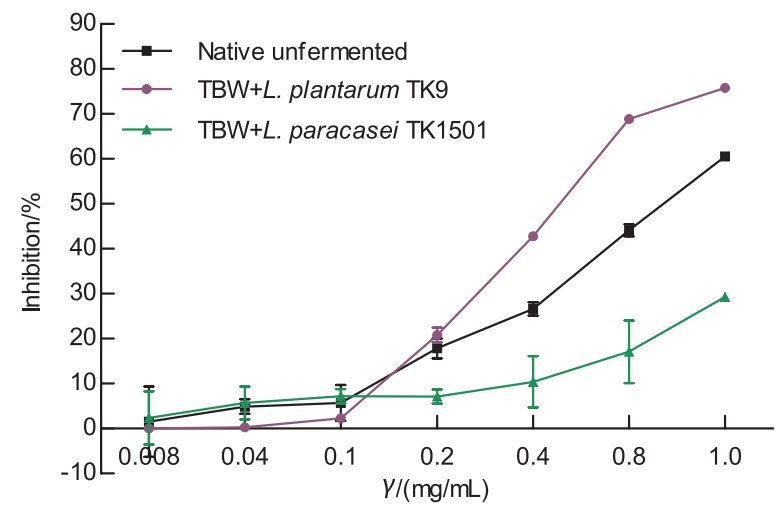

Fig. 1. a-Glucosidase inhibition activity by Tartary buckwheat (TBW) ethanol extracts obtained in solid-state fermentation. Each value corresponds to the mean of three independent replicates with error bars indicating the standard deviations 
was even less effective, with respective inhibitory activities of $(2.7 \pm 5.9),(5.7 \pm 3.7),(7.2 \pm 1.6),(7.1 \pm 1.6),(10.4 \pm 5.7),(17.1 \pm 7.0)$ and $(29.2 \pm 0.5) \%$, with an $I C_{50}$ value $>1 \mathrm{mg} / \mathrm{mL}$. The $I C_{50}$ value of the positive control (acarbose) for a-glucosidase inhibition was $0.85 \mathrm{mg} / \mathrm{mL}$ (26). Earlier studies have reported that Tartary buckwheat inhibits a-glucosidase (29). Based on the relationship between bioactive components and a-glucosidase inhibition, TFC and TPC are considered to play an important role in the inhibitory activity of Tartary buckwheat (21). Some reports have suggested that many flavonoids can inhibit a-glucosidase activity (30). Moreover, quercetin has a higher inhibitory activity on a-glucosidase than rutin (4). Therefore, we suspected that the L. plantarum TK9 fermentation enhanced the quercetin content. It has already been confirmed that certain strains of Lactobacillus have inhibitory potential against a range of a-glucosidases and at least one $\beta$-glucosidase (31). It was found that a product fermented by L. plantarum TK9 has a significantly higher inhibitory effect on a-glucosidase than that by L. paracaseiTK1501. The inhibition of intestinal a-glucosidase is a known strategy to regulate blood glucose (32). Although synthetic inhibitors of intestinal a-glucosidase have found wide application in the clinic, there are considerations concerning cost and side effects. Consequently, there is a demand for alternatives from natural plant, animal and probiotic sources (33-35), and many bacteria have been shown to have inhibitory activity. Cell-free supernatants of six strains of $L$. plantarum showed inhibitory activity ranging from 24.96 to $41.81 \%$ (36). Notably, the strain TK9 investigated in this study was even more active than these six strains. Its comparatively high inhibitory activity suggests that Tartary buckwheat fermented by L. plantarum TK9 may have dual beneficial effects on glycaemia regulation by reducing the intestinal absorption of carbohydrates. Similarly, reports have shown that L. plantarum NCU116 and carrot juice fermented by it had the potential to regulate blood glucose levels (37). The Tartary buckwheat fermented by L. plantarum TK9 had very satisfactory a-glucosidase inhibitory activity, and it merits commercial exploration as an efficient agent for the management of glucose metabolism.

\section{Inhibition of DPP-IV activity}

Dipeptidyl peptidase IV (DPP-IV) is a highly specialized aminopeptidase that appears to be a major physiological modulator of a number of regulatory peptides, neuropeptides and chemokines $(38,39)$. GLP-1 is a gastrointestinal hormone that can reduce appetite, suppress glucagon secretion, stimulate insulin secretion and reduce gastric emptying (13). Reports show that GLP-1 levels reduced after a mixed meal and an oral glucose load in patients with diabetes type 2 (40). The enzyme DPP-IV rapidly metabolizes GLP-1 (41). Preliminary clinical data have shown the potential of DPP-IV inhibitors in treating diabetes type 2 (42). The need to develop safe DPP-IV inhibitors has led to increased attention to natural sources. Recently, natural sources as diverse as dietary proteins, medicinal plants, and marine life have been confirmed to have DPP-IV inhibitory effects (43). All extracts used in our research showed DPP-IV inhibitory activity in a concentration-dependent manner. The Tartary buckwheat extracts ( $4 \mathrm{mg} / \mathrm{mL}$ ) showed DPP-IV inhibitory potential, among which the extract of Tartary buckwheat fermented by L. paracaseiTK1501 displayed the greatest inhibition $(77.2 \pm 1.9) \%$, followed by L. plantarum TK9 $(57.3 \pm 2.1) \%$ and native non-fermented Tartary buckwheat extract (39.9 \pm 1.4$) \%$. Fig. 2 shows the inhibitory effects of the three different Tartary buckwheat samples (native, fermented by L. plantarum TK9 and L. paracasei TK1501) at different concentrations $(0.2-5 \mathrm{mg} / \mathrm{mL})$, with $\mathrm{IC}_{50}>5,3.45$ and $2.47 \mathrm{mg} / \mathrm{mL}$, respectively. Unlike the results of a-glucosidase inhibition, the extract of Tartary buckwheat fermented by L. paracasei TK1501 showed the best inhibition of DPP-IV activity. The positive control diprotin A had an $\mathrm{IC}_{50}$ of $1.15 \mathrm{mg} / \mathrm{mL}$, and behaved as a competitive inhibitor, which was in agreement with the literature $(12,21)$. Our work represents the first report on the inhibition of DPP-IV activity by Tartary buckwheat. We speculated that effective inhibitory components might not be related to TPC or TFC in view of our previous results. Similarly, some reports suggest that the strains themselves were the most likely producer of the inhibitory compounds $(1,12,21,44)$. The results of this study suggest that fermented Tartary buckwheat has antihyperglycaemic properties, and hence might provide a new dietotherapy food for the control of diabetes.

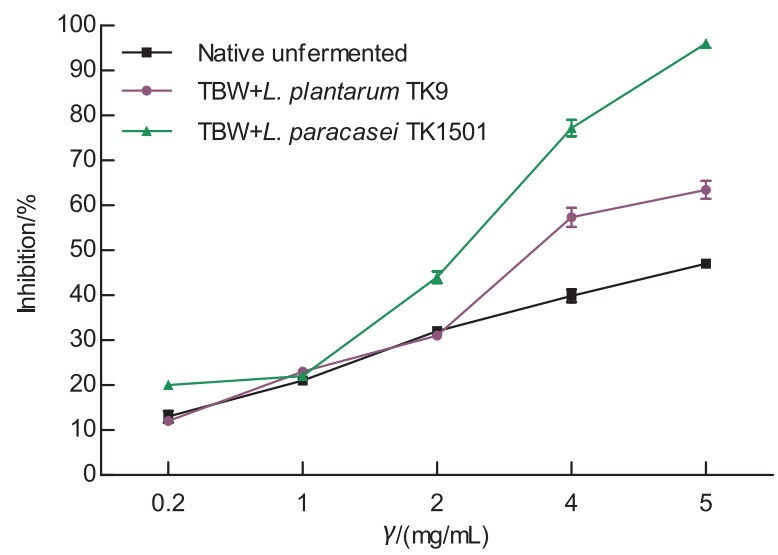

Fig. 2. Dipeptidyl peptidase IV (DPP-IV) inhibition activity by Tartary buckwheat (TBW) ethanol extracts obtained in solid-state fermentation (SSF). Each value corresponds to the mean of three independent replicates with error bars indicating the standard deviations

\section{CONCLUSIONS}

This study evaluated the chemical composition and antioxidant and antihyperglycaemic activities of two Tartary buckwheat samples fermented using pure cultures of Lactobacillus plantarum TK9 and L. paracasei TK1501, respectively, and compared them to native unfermented Tartary buckwheat. There was little difference in the total phenolic and total flavonoid contents between the fermented and native unfermented Tartary buckwheat. Moreover, the extracts of Tartary buckwheat fermented by L. plantarum TK9 and L. paracasei TK1501 showed remarkable a-glucosidase and dipeptidyl peptidase IV inhibitory effects, respectively. Taken together, the data 
indicate good potential of fermented Tartary buckwheat for application in the production of antidiabetic functional foods.

\section{ACKNOWLEDGEMENTS}

The authors wish to acknowledge the financial support provided by Tianjin University of Science and Technology Research Fund Project (No. 20130111), the Tianjin Social Science Planning Program (grant number TJGL15-033) and the Innovative Research Teams of Tianjin Municipal Education Commission project (No. TD13-5015).

\section{REFERENCES}

1. Imamura K, Tsuyama Y, Hirata T. Identification and characterization of a novel fermented substance produced by edible Aspergillus oryzae AO-1 that inhibits DPP-IV activity. J Biosci Bioeng. 2011;111(1):37-40.

https://doi.org/10.1016/j.jbiosc.2010.08.013

2. Zimmet P, Alberti KGMM, Shaw J. Global and societal implications of the diabetes epidemic. Nature. 2001;414:782-7. https://doi.org/10.1038/414782a

3. Sun T, Ho CT. Antioxidant activities of buckwheat extracts. Food Chem. 2005;90(4):743-9. https://doi.org/10.1016/j.foodchem.2004.04.035

4. Lukšič L, Bonafaccia G, Timoracka M, Vollmannova A, Trček $J$, Koželj Nyambe T, et al. Rutin and quercetin transformation during preparation of buckwheat sourdough bread. J Cereal Sci. 2016;69:71-6. https://doi.org/10.1016/j.jcs.2016.02.011

5. Zhu F. Chemical composition and health effects of Tartary buckwheat. Food Chem. 2016;203:231-45. https://doi.org/10.1016/j.foodchem.2016.02.050

6. Bonafaccia G, Marocchini M, Kreft I. Composition and technological properties of the flour and bran from common and tartary buckwheat. Food Chem. 2003;80(1):9-15. https://doi.org/10.1016/S0308-8146(02)00228-5

7. Guo XD, Wu CS, Ma YJ, Parry J, Xu YY, Liu H, Wang M. Comparison of milling fractions of tartary buckwheat for their phenolics and antioxidant properties. Food Res Int. 2012 49(1):53-9.

https://doi.org/10.1016/j.foodres.2012.07.019

8. Qin P, Wu L, Yao Y, Ren G. Changes in phytochemical compositions, antioxidant and a-glucosidase inhibitory activities during the processing of tartary buckwheat tea. Food Res Int. 2013;50(2):562-7.

https://doi.org/10.1016/j.foodres.2011.03.028

9. Ren Q, Wu C, Ren Y, Zhang J. Characterization and identification of the chemical constituents from tartary buckwheat (Fagopyrum tataricum Gaertn) by high performance liquid chromatography/photodiode array detector/linear ion trap FTICR hybrid mass spectrometry. Food Chem. 2013;136(34):1377-89.

https://doi.org/10.1016/j.foodchem.2012.09.052
10. Genet S, Kale RK, Baquer NZ. Alterations in antioxidant enzymes and oxidative damage in experimental diabetic rat tissues: Effect of vanadate and fenugreek (Trigonella foenum graecum). Mol Cell Biochem. 2002;236(1-2):7-12. https://doi.org/10.1023/A:1016103131408

11. Mazziotti G, Gazzaruso C, Giustina A. Diabetes in Cushing syndrome: Basic and clinical aspects. Trends Endocrinol Metab. 2011;22(12):499-506.

https://doi.org/10.1016/j.tem.2011.09.001

12. Zeng Z, Luo J, Zuo F, Zhang Y, Ma H, Chen S. Screening for potential novel probiotic Lactobacillus strains based on high dipeptidyl peptidase IV and a-glucosidase inhibitory activity. J Funct Foods. 2016;20:486-95. https://doi.org/10.1016/j.jff.2015.11.030

13. Drucker DJ, Nauck MA. The incretin system: Glucagon-like peptide-1 receptor agonists and dipeptidyl peptidase-4 inhibitors in type 2 diabetes. Lancet. 2006;368(9548):1696-705. https://doi.org/10.1016/S0140-6736(06)69705-5

14. Sales PM, Souza PM, Simeoni LA, Magalhães PO, Silveira D. a-Amylase inhibitors: A review of raw material and isolated compounds from plant source. J Pharm Pharm Sci. 2012;15(1):141-83.

https://doi.org/10.18433/J35S3K

15. Reimer MK, Holst JJ, Ahrén B. Long-term inhibition of dipeptidyl peptidase IV improves glucose tolerance and preserves islet function in mice. Eur J Endocrinol. 2002;146:717-27. https://doi.org/10.1530/eje.0.1460717

16. Wang HK, Ng YK, Koh E, Yao L, Chien AS, Lin HX, Lee YK. RNA-Seq reveals transcriptomic interactions of Bacillus subtilis natto and Bifidobacterium animalis subsp. lactis in whole soybean solid-state co-fermentation. Food Microbiol. 2015;51:25-32. https://doi.org/10.1016/j.fm.2015.04.012

17. IBM SPSS Statistics, v. 22.0, IBM, Armonk, NY, USA; 2013. Available from: http://www-01.ibm.com/support/docview. wss?uid=swg21646821.

18. Zhang N, Li D, Zhang X, Shi Y, Wang H. Solid-state fermentation of whole oats to yield a synbiotic food rich in lactic acid bacteria and prebiotics. Food Funct. 2015;6(8):2620-5. https://doi.org/10.1039/C5FO00411J

19. Singleton VL, Rossi JA. Colorimetry of total phenolics with phosphomolybdic-phosphotungstic acid reagents. Am J Enol Vitic. 1965;16(3):144-58.

20. Xiong HB, Liu MH, Chen MH, Dai JH, Gao YT. Antioxidant activity and flavones compounds contents of tartary buckwheat seedlings extract. Asian J Chem. 2013;25(8):4189-94. https://doi.org/10.14233/ajchem.2013.13889

21. Ran JX, MaTJ. Effect of total flavonoid and total polyphenol from Tartary buckwheat sprouts and their inhibitor activities on a-glycosidase. 2016 Eighth International Conference on Measuring Technology and Mechatronics Automation (ICMTMA); 2016 March 11-12; Macau; 2016. pp. 258-61. https://doi.org/10.1109/ICMTMA.2016.70 
22. Đorđević TM, Šiler-Marinković SS, Dimitrijević-Branković SI. Effect of fermentation on antioxidant properties of some cereals and pseudo cereals. Food Chem. 2010;119(3):957-63. https://doi.org/10.1016/j.foodchem.2009.07.049

23. DulfFV, Vodnar DC, Socaciu C. Effects of solid-state fermentation with two filamentous fungi on the total phenolic contents, flavonoids, antioxidant activities and lipid fractions of plum fruit (Prunus domestica L.) by-products. Food Chem. 2016;209:27-36. https://doi.org/10.1016/j.foodchem.2016.04.016

24. Oduguwa OO, Edema MO, Ayeni AO. Physico-chemical and microbiological analyses of fermented corn cob, rice bran and cowpea husk for use in composite rabbit feed. Bioresour Technol. 2008;99(6):1816-20.

https://doi.org/10.1016/j.biortech. 2007.03.036

25. Chien HL, Huang HY, Chou CC. Transformation of isoflavone phytoestrogens during the fermentation of soymilk with lactic acid bacteria and bifidobacteria. Food Microbiol. 2006;23(8):772-8. https://doi.org/10.1016/j.fm.2006.01.002

26. Shukla S, Park J, Kim DH, Hong SY, Lee JS, Kim M. Total phenolic content, antioxidant, tyrosinase and a-glucosidase inhibitory activities of water soluble extracts of noble starter culture Doenjang, a Korean fermented soybean sauce variety. Food Control. 2016;59:854-61.

https://doi.org/10.1016/j.foodcont.2015.07.003

27. Horbowicz M, Wiczkowski W, Szawara-Nowak D, Sawicki T, Kosson R, Sytykiewicz H. The level of flavonoids and amines in de-etiolated and methyl jasmonate treated seedling of common buckwheat. Phytochem Lett. 2015;13:15-9.

https://doi.org/10.1016/j.phytol.2015.05.011

28. Bolen S, Feldman L, Vassy J, Wilson L, Hsin-Chieh Y, Marinopoulos S, et al. Systematic review: Comparative effectiveness and safety of oral medications for type 2 diabetes mellitus. Ann Intern Med. 2007;147(6):386-99

29. Li SQ, Zhang QH. Advances in the development of functional foods from buckwheat. Crit Rev Food Sci Nutr. 2001;41 (6):451-64. https://doi.org/10.1080/20014091091887

30. Tadera K, Minami Y, Takamatsu K, Matsuoka T. Inhibition of a-glucosidase and a-amylase by flavonoids. J Nutr Sci Vitaminol. 2006;52(2):149-53. https://doi.org/10.3177/jnsv.52.149

31. Panwar H, Calderwood D, Grant IR, Grover S, Green BD. Lactobacillus strains isolated from infant faeces possess potent inhibitory activity against intestinal alpha- and beta-glucosidases suggesting anti-diabetic potential. Eur J Nutr. 2014; 53(7):1465-74.

https://doi.org/10.1007/s00394-013-0649-9

32. Lebovitz HE. Alpha-glucosidase inhibitors. Endocrinol Metab Clin North Am. 1997;26(3):539-51. https://doi.org/10.1016/S0889-8529(05)70266-8
33. Tundis R, Loizzo MR, Menichini F. Natural products as a-amylase and a-glucosidase inhibitors and their hypoglycaemic potential in the treatment of diabetes: An update. Mini Rev Med Chem. 2010;10(4):315-31.

https://doi.org/10.2174/138955710791331007

34. Katekhaye SD, Nagmoti DM. a-glucosidase and a-amylase inhibition activities of pithecellobium dulce bark and leaves. Phytopharmacology. 2013;4(1):123-30.

35. Yamada T, Hida H, Yamada Y. Chemistry, physiological properties, and microbial production of hydroxycitric acid. Appl Microbiol Biotechnol. 2007;75(5):977-82.

https://doi.org/10.1007/s00253-007-0962-4

36. Li X, Wang N, Yin B, Fang D, Zhao J, Zhang H, et al. Lactobacillus plantarum $\mathrm{X} 1$ with a-glucosidase inhibitory activity ameliorates type 2 diabetes in mice. RSC Adv. 2016;6(68): 63536-47.

https://doi.org/10.1039/C6RA10858J

37. Li C, Ding Q, Nie SP, Zhang YS, Xiong T, Xie MY. Carrot juice fermented with Lactobacillus plantarum NCU116 ameliorates type 2 diabetes in rats. J Agric Food Chem. 2014; 62:11884-91.

https://doi.org/10.1021/jf503681r

38. Mentlein R. Dipeptidyl-peptidase IV (CD26)-role in the inactivation of regulatory peptides. Regul Peptides. 1999;85 (1):9-24.

https://doi.org/10.1016/S0167-0115(99)00089-0

39. Mentlein R. Proline residues in the maturation and degradation of peptide hormones and neuropeptides. FEBS Lett. 1988;234(2):251-6. https://doi.org/10.1016/0014-5793(88)80092-9

40. Rotella CM, Pala L, Mannucci E. Glucagon-like peptide 1 (GLP-1) and metabolic diseases. J Endocrinol Invest. 2005; 28(10):746-58.

https://doi.org/10.1007/BF03347560

41. Holst JJ. Treatment of type 2 diabetes mellitus with agonists of the GLP-1 receptor or DPP-IV inhibitors. Expert Opin Emerg Drugs. 2004;9(1):155-66.

https://doi.org/10.1517/14728214.9.1.155

42. Nielsen LL. Incretin mimetics and DPP-IV inhibitors for the treatment of type 2 diabetes. Drug Discov Today. 2005; 10(10):703-10.

https://doi.org/10.1016/S1359-6446(05)03460-4

43. Gao Y, Zhang Y, Zhu J, Li B, Li Z, ZhuW, et al. Recent progress in natural products as DPP-4 inhibitors. Future Med Chem. 2015;7(8):1079-89. https://doi.org/10.4155/fmc.15.49

44. Zeng Z, Luo JY, Zuo FL, Yu R, Zhang Y, Ma HQ, Chen SW. Bifidobacteria possess inhibitory activity against dipeptidyl peptidase-IV. Lett Appl Microbiol. 2016;62(3):250-5.

https://doi.org/10.1111/lam.12510 